\title{
Pulmonary artery hemodynamic assessment of blood flow characteristics in repaired tetralogy of Fallot patients versus healthy child volunteers
}

\author{
Liwei Hu ${ }^{1 \#}$, Rongzhen Ouyang ${ }^{1 \#}$, Aimin Sun ${ }^{1}$, Qian Wang ${ }^{1}$, Chen Guo ${ }^{1}$, Yafeng Peng ${ }^{1}$, Yan Qin ${ }^{2}$, \\ Yong Zhang ${ }^{3}$, Yang Xiang ${ }^{4}$, Yumin Zhong ${ }^{1}$
}

${ }^{1}$ Diagnostic Imaging Center, ${ }^{2}$ Department of Cardiovascular and Thoracic Surgery, Shanghai Children's Medical Center Affiliated with Shanghai Jiao Tong University School of Medicine, Shanghai 200127, China; ${ }^{3}$ MR research, GE Healthcare, Shanghai 201203, China; ${ }^{4}$ J.C. Wu Center for Aerodynamics, School of Aeronautics and Astronautics, Shanghai Jiao Tong University, Shanghai 200240, China

\#These authors contributed equally to this work as co-first authors.

Correspondence to: Yumin Zhong; Rongzhen Ouyang. No. 1678 Dong Fang Road, Shanghai 200127, China. Email: zyumin2002@163.com; ouyangrongzhen@scmc.com.cn.

Background: This study aimed to assess the severity of helix and vortex flow in pulmonary artery hemodynamic using 4-dimensional flow cardiac magnetic resonance (4D flow CMR) in patients with repaired tetralogy of Fallot (rTOF) and healthy child volunteers and to explore the relationship between pulmonary hemodynamic changes and right heart function.

Methods: CMR studies were performed in 25 rTOF patients (15 M/10 F; 8.44 4.52 years) and 10 normal child volunteers (7 M/3 F; $8.2 \pm 1.22$ years) on 3.0T MR scanners. Cardiac function was calculated in the patient and control groups. Systolic diameter, peak velocity, net flow, and regurgitation was quantified in the main pulmonary artery (MPA) plane, left pulmonary artery (LPA) plane, and right pulmonary artery (RPA) plane. The relationship between the hemodynamic parameters and quantitative flow indices and right ventricular (RV) function were analyzed through simple linear regression analysis using Pearson R-values. We analyzed differences in flow patterns between the 2 groups for the same slice. According to the severity of the helix and vortex flow in the 4D flow CMR, we categorized rTOF patients into the following groups: group 1, severe flow grading; group 2, mild flow grading; group 3, no flow grading; the control cases with no flow grade were included in group 4. We compared RV cardiac function, wall shear stress (WSS), and viscous energy loss (EL) between group 1+2 and group 3+4 using unpaired $t$-test analysis for normally distributed data and the Mann-Whitney test for non-normally distributed continuous variables.

Results: RV end-diastolic volume index $\left(\mathrm{EDV}_{\mathrm{i}}\right)(127.8 \pm 36.13$ vs. 83.11 \pm 6.18 , respectively; $\mathrm{P}<0.001)$, $\mathrm{RV}$ end-systolic volume index $\left(\mathrm{ESV}_{\mathrm{i}}\right)(65.14 \pm 27.02$ vs. 36.13 \pm 5.95 , respectively; $\mathrm{P}<0.001)$, and ejection fraction (EF) $(49.97 \pm 6.39$ vs. $56.71 \pm 4.56$, respectively; $\mathrm{P}=0.006$, ) were significantly different between the groups. The rTOF diameters of the MPA and RPA were significantly larger than those of the control group (19.74 \pm 4.01 vs. $14.97 \pm 2.37$ for MPA, $\mathrm{P}=0.001 ; 12.04 \pm 3.28$ vs. $8.99 \pm 1.23$ for $\mathrm{RPA}, \mathrm{P}=0.004$, respectively). There were correlations between peak WSS and pulmonary regurgitation $(\mathrm{PR})$ in the MPA $(\mathrm{R}=0.48$, $\mathrm{P}=0.014)$, correlations between peak systolic $\mathrm{EL}$ and $\mathrm{RVEDV}(\mathrm{R}=0.51, \mathrm{P}=0.008)$, and between peak systolic EL and RVESV ( $\mathrm{R}=0.51, \mathrm{P}=0.009)$. The peak systole and diastole WSS of group $1+2$ were significantly different compared to group $3+4$ in the MPA $(\mathrm{P}<0.05)$. The peak systole and diastole EL of group $1+2$ was significantly different from group $3+4$ in the MPA $(\mathrm{P}<0.05)$. The peak systole EL of group $1+2$ was significantly different from group $3+4$ in the RPA $(\mathrm{P}<0.01)$.

Conclusions: Increased peak WSS and EL were associated with pulmonary hemodynamic changes in the MPA and RPA. There might be an earlier marker of evolving hemodynamic inefficiency than that in traditional parameters. The better understanding of pulmonary artery hemodynamic assessment in rTOF may lead to a greater insight into pulmonary artery (PA)-RV interactions and how they ultimately impact RV function. 


\begin{abstract}
Keywords: 4D flow; cardiovascular magnetic resonance (CMR); tetralogy of Fallot (TOF); wall shear stress;
\end{abstract} viscous energy loss

Submitted Jun 12, 2019. Accepted for publication Mar 24, 2020.

doi: $10.21037 /$ qims.2020.03.23

View this article at: http://dx.doi.org/10.21037/qims.2020.03.23

\section{Introduction}

Tetralogy of Fallot (TOF) is one of the most common complex congenital heart defects requiring repair in infancy, which consists of malalignment ventricular septal defect, pulmonary stenosis, overriding aortic root, and right ventricular (RV) hypertrophy (1). With the introduction of modern surgical techniques, a dramatic $40 \%$ reduction in deaths associated with TOF was noted from 1979 to 2005, with most patients now surviving well into adulthood (2). Children with TOF require frequent and long-term surveillance for common postoperative sequelae, including for residual $\mathrm{RV}$ outflow tract obstruction, pulmonary regurgitation (PR), RV dilatation or hypertrophy, and right or left ventricular dysfunction (3).

Cardiovascular magnetic resonance (CMR) is routinely used to assess the RV size and function, along with PR. Four-dimensional flow (4D flow) magnetic resonance imaging (MRI) provides hemodynamic information, allowing for flow quantification, assessment of complex flow patterns such as helical or vortical flow, and quantification of higher-order fluid dynamic metrics such as wall shear stress (WSS) (4), turbulent kinetic energy (5), and viscous energy loss (EL) (6). Recently, some authors reported that kinetic energy is a potential early marker of RV efficiency to guide intervention in repaired tetralogy of Fallot (rTOF) patients $(7,8)$. Due to the anatomy of rTOF, the blood flow in the pulmonary artery is known to be complex and can exhibit a swirling, or helical flow pattern. Geiger et al. (9) reported marked variation in blood flow in all rTOF patients identified, including severe vortex formation and retrograde flow in the RV outflow tract and pulmonary arteries.

To our knowledge, there are few current methods that evaluate the pulmonary artery by hemodynamics indices using a 4D flow sequence in rTOF patients. Some recent research exists, but this literature only characterizes abnormal flow patterns (9) or focuses on the comparison of 2D and 4D flow MRI for flow quantification $(10,11)$. Therefore, this study aimed to (I) assess the severity of helix and vortex in the main pulmonary artery (MPA), right pulmonary artery (RPA), and left pulmonary artery (LPA) by 4D flow CMR in patients with rTOF and child volunteers. (II) The relationship between pulmonary hemodynamic changes and right heart function was also explored.

\section{Methods}

\section{Subjects}

This single-center retrospective cohort study was approved by the hospital institutional review board. In total, 25 patients with rTOF (gender $15 \mathrm{M} / 10 \mathrm{~F}$; age $8.44 \pm 4.52$ years) and 10 healthy child volunteers (gender $7 \mathrm{M} / 3 \mathrm{~F}$; age $8.2 \pm 1.22$ years) were included in this study as a control group. The exclusion criteria were as follows: (I) residual shunt and residual obstruction examined by echocardiography in the rTOF group; (II) moderate to severe stenosis ( $>300-400 \mathrm{~cm} / \mathrm{s}$ ) of the left and RPA during echocardiography follow-up; and (III) patients with inadequate image quality (aliasing, inhomogeneous magnetic field, motion artifact, imaging noising, etc.) for the cardiac function or hemodynamic analysis. The age at CMR, heart rate, body surface area, the surgical procedures, and follow-up times after surgery were collected. Patients were examined with contrast agents and informed consent was signed by participant's parents or legal guardians.

The control population included aged- and gender-matched children with typical intracardiac structure, ventricular function, and no history of cardiothoracic surgery, which were ruled out by echocardiography. The control group underwent CMR without contrast agent and informed consent was signed by the participant's parents or legal guardians.

\section{Cardiac magnetic resonance}

All subjects underwent 2D balanced steady-state free precession (2D b-SSFP) cine sequence performed with a 3.0T scanner (MR750, GE Healthcare, Waukesha, WI, USA) by short-axis view covering the right ventricle to measure cardiac function. The following MR imaging 

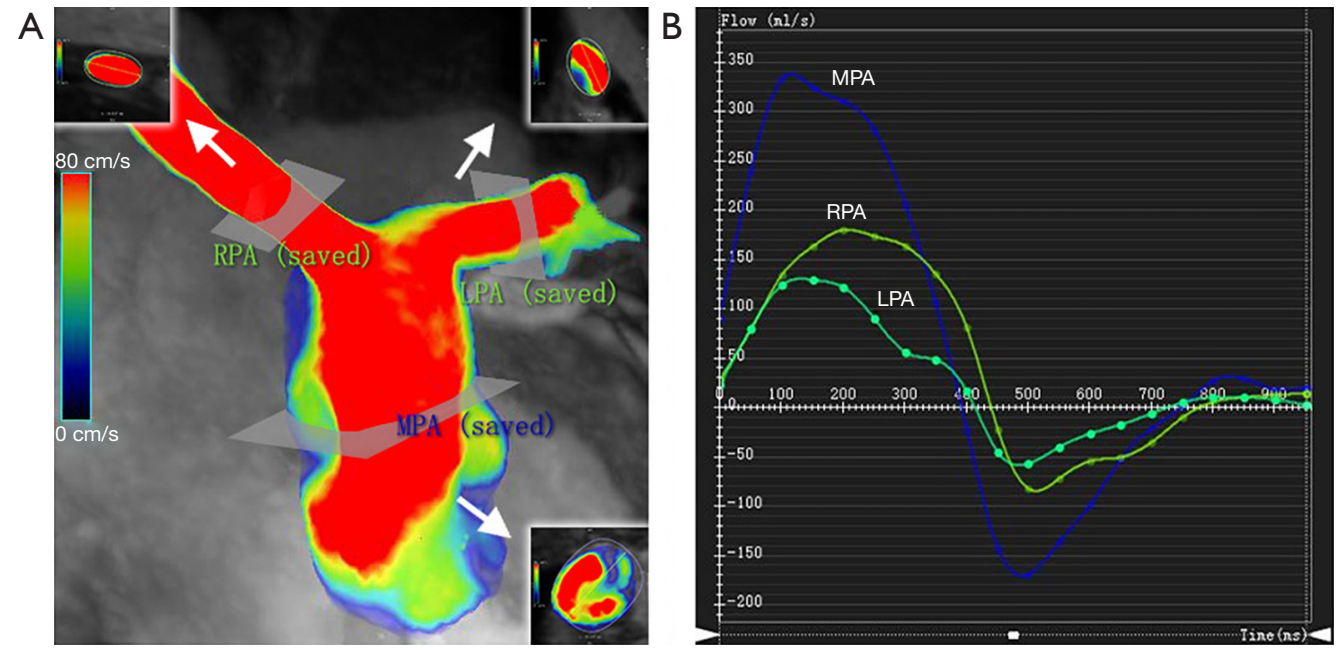

Figure 1 Routine blood flow parameters were analyzed in the pulmonary artery. (A) Locations of the MPA, RPA, and LPA cutplanes, which were placed approximately 10-15 mm downstream from the pulmonary valve or LPA/RPA bifurcation. (B) The results of peak velocity in MPA, RPA, and LPA cutplanes. MPA, main pulmonary artery; RPA, right pulmonary artery; LPA, left pulmonary artery. Arrows indicate sliced sheet of pulmonary artery.

parameters were used: repetition time ms/echo time $\mathrm{ms}$, 3.1/1.5; acquisition matrix, 288-320×288-320; flip angle, $60^{\circ}$; interslice gap $0 \mathrm{~mm}$; section thickness, $6-7 \mathrm{~mm}$. Finally, 20-30 phases per cardiac cycle were reconstructed.

The CMR 4D flow data were acquired after the bolus injection of $0.1-0.2 \mathrm{mmol} / \mathrm{kg}$ gadolinium-based contrast agent in rTOF subjects. 4D flow sequence CMR was used to characterize and quantify flow hemodynamics in the whole heart. Data sets were acquired with prospective electrocardiographic (ECG)-gating during free-breathing. The image acquisition volume was in the coronal plane with full volumetric coverage of the great arteries. Sequence parameters were as follows: echo time, $2.1 \mathrm{~ms}$; repetition time, $4.3 \mathrm{~ms}$; flip angle $8-12^{\circ}$; voxel size, $(1.2$ to 1.6$) \times(1.2$ to 1.6$) \times(1.2$ to 1.6$) \mathrm{mm}^{3}$; temporal resolution, $34.4 \mathrm{~ms}$; velocity sensitivity, $160-200 \mathrm{~cm} / \mathrm{s}$, parallel imaging with reduction factor, $\mathrm{R}=2$. The resulting scan time was on the order of 10-12 minutes. We followed the parameter settings of the protocol according to the 4D Flow CMR consensus statement (12). Due to the smaller field of vision (FOV), a higher spatial resolution is recommended for a pediatric population (13). We selected a thinner slice thickness to achieve isotropic voxels. The velocity encoding range was determined by referring to peak velocities of the same vessels in echocardiography. Optimization of flow parameters was able to fulfill flow quantification in $4 \mathrm{D}$ flow CMR covering the heart and pulmonary artery.

\section{$R V$ function analysis}

Quantitative analysis of cardiac function was measured by 2D b-SSFP in rTOF subjects and the control group using CVI version 5.9.1 software (Circle Cardiovascular Imaging, Calgary, AB, Canada). The endocardial and epicardial borders of the right ventricle were semi-auto traced on short-axis cine images at end-systole and end-diastole. The right ventricle end-diastolic volume (EDV) and endsystolic volume (ESV) were obtained from short-axis stacks. Ejection fraction (EF) was calculated automatically. Height and weight were measured, and body surface area (BSA) was calculated. Ventricular volume was indexed to the BSA.

\section{D flow data processing and analysis}

The number of reconstructed temporal frames was limited to 20 or 30 frames. 4D flow MRI data were preprocessed to correction using CVI version 5.9.1 software (Circle Cardiovascular Imaging, Calgary, AB, Canada). During visualization with CVI, the shaded surface display was created based on 4D flow data. The central line segment was used to develop a geometric structure of the main, left, and right pulmonary artery (MPA, RPA, and LPA, respectively). Analysis planes were semi-automatically placed orthogonal to the anticipated main low direction (Figure 1) in the MPA, RPA, and LPA. The imaging was required to have a line of at least 4 pixels (i.e., 16 voxels covering the pulmonary 

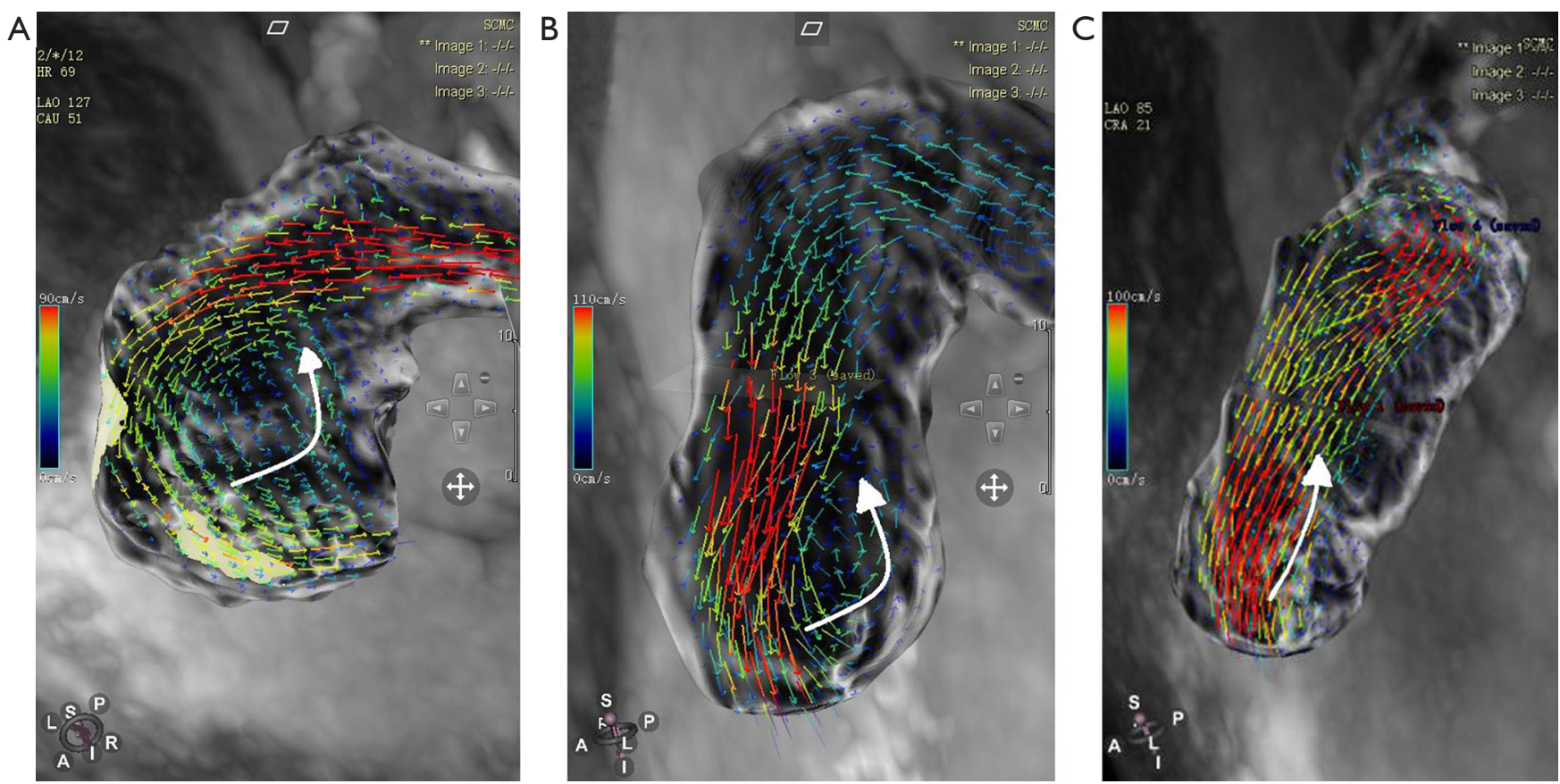

Figure 2 Vortex severity was graded in the rTOF patients. (A) Follow-up magnetic resonance imaging of a 12-year-old, female child using a 4D flow sequence. During diastole, the flow pattern of an rTOF patient with severe PA dilation can be seen (group 1). A severe vortex formed in the PA, and the flow velocities were lower; the vortex formed by regurgitation was close to the pulmonary valve. (B) Follow-up magnetic resonance imaging of a 9-year-old, male child using 4D flow sequence. During diastole, a flow pattern of an rTOF patient with PA was observed, and the vortices formed in the PA and mild vortex locally affected the wall shear stress inside the vessel (group 2). A vortex formed by regurgitation found close to the pulmonary valve. (C) Follow-up magnetic resonance imaging of a 15-year-old, male teenager using 4D flow sequence. During diastole, none-flow grading appeared in the pulmonary artery (group 3). rTOF, repaired tetralogy of Fallot; PA, pulmonary artery. Curved arrows indicate direction of blood flow.

arteries) along the diameter of each vessel flow pattern analysis. 4D-blood flow visualization was evaluated for each patient and volunteer in consensus reading with regard to vortex formation and helical flow using streamlines. Time-resolved $3 \mathrm{D}$ velocity vector mapping was viewed dynamically and rotated or magnified for inspection in any chosen orientation by 2 observers (both with more than 3 years of experience in 4D flow image processing). The velocity vector represents the rate of change of the position of an object. As illustrated in Figure 2, vortices were defined as regional circular flow patterns deviating by more than $90^{\circ}$ from the physiological flow direction along the vessel lumen (9). Vortex severity was graded into 3 categories by Bürk et al., who described the strength of prominent secondary flow formations as follows (14): no vortex formation $\left(0^{\circ}<\right.$ flow rotation $\left.<180^{\circ}\right)=0$, moderate vortex $\left(180^{\circ}<\right.$ flow rotation $\left.<360^{\circ}\right)=1$, pronounced vortex (flow rotation $>360^{\circ}$ ) $=2$. As illustrated in Figure 3, helical flow of $\mathrm{PA}$ was defined as its tangent making a constant angle with a fixed line in space (15). Helical flow in the RV outflow tract, and in the right and left pulmonary arteries was evaluated using the following a 3-grade ranking: no helical flow $=0$, moderate helical flow (flow rotation $\angle 360^{\circ}$ ) $=1$, severe helical flow (flow rotation $>360^{\circ}$ ) $=2$. If patients simultaneously had different types of flow grading, the higher-grade flow prevailed. The groups were defined as follows: group 1, the repair TOF cases with severe flow grading, including MPA, RPA, and LPA; group 2, the repair TOF cases of moderate flow grading, including MPA, RPA, and LPA; group 3, the repair TOF cases of none flow grading, including MPA, RPA, and LPA; group 4, no flow grading in controls.

Additionally, quantitative flow analysis was performed with previously described software. Systolic diameter, peak velocity, net flow, and PR fraction were measured in the MPA, RPA, and LPA of both groups (Figure 1). WSS was calculated from $3 \mathrm{D}$ velocity vector fields, as described by Stalder et al. (16). Viscous EL was calculated as described by Barker et al. (6). WSS and EL were calculated from the 

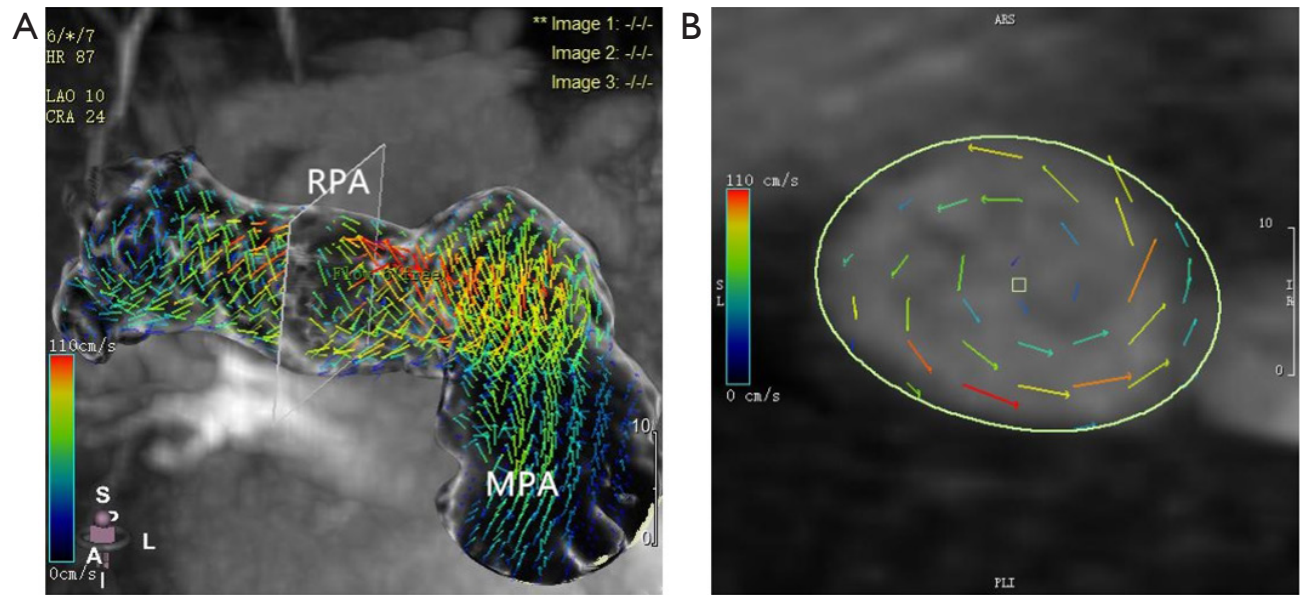

Figure 3 Follow-up magnetic resonance imaging of a 5-year-old, male child using 4D flow sequence. (A) During systole, RPA hemodynamic vectors were analyzed in 3 dimensions inducing opposite-sign wall helical flow (flow rotation $>360^{\circ}$ ). (B) During systole, the flow characteristics were related to the anatomy of the heart and the main blood flow directions in RPA cutplane. Blood flow vector distribution showing abnormal flow along the tangent direction of the artery wall. RPA, right pulmonary artery.

peak-systolic and end-diastolic velocities.

\section{Statistical analysis}

All data were presented as means \pm standard deviations or numbers (percentage) and ranges. A comparison of cardiac function and blood flow parameters between rTOF patients and volunteers was performed using the unpaired $t$-test for normally distributed variables and the Mann-Whitney test for non-normally distributed variables. Comparison occurrence frequency of normal and abnormal flow patterns was also performed in the pulmonary artery between the 2 groups. The relationship between the hemodynamic parameters and quantitative flow indices and RV function were analyzed through simple linear regression analysis using Pearson R-values. To compare RV cardiac function, WSS, and viscous EL between group $1+2$ and group $3+4$ unpaired $t$-test analysis for normally distributed data and Mann-Whitney test for non-normally distributed continuous variable were used. The null hypothesis was rejected for P-values less than 0.05 .

\section{Results}

\section{Clinical information}

In rTOF and control groups, the mean age at CMR was $8.44 \pm 4.52$ and $8.2 \pm 1.22$ years, respectively. In 25 rTOF patients, 2 types of surgical procedures were identified: partial valve preserving $(\mathrm{n}=2,8 \%)$ and transannular patch $(\mathrm{n}=23,92 \%)$. The mean follow-up time was $7.24 \pm 4.25$ years. The mean heart rate and body surface area are shown in Table 1.

\section{Cardiac function and flow quantification}

Right ventricle function was significantly different between the rTOF and control group in terms of RV enddiastolic volume index $\left(\mathrm{EDV}_{\mathrm{i}}\right)(127.8 \pm 36.13$ vs. 83.11 \pm 6.18 , respectively; $\mathrm{P}<0.001), \mathrm{RV}$ end-systolic volume index $\left(\mathrm{ESV}_{\mathrm{i}}\right)$ (65.14 \pm 27.02 vs. $36.13 \pm 5.95$, respectively; $\mathrm{P}<0.001)$, and $\mathrm{EF}$ (49.97 \pm 6.39 vs. $56.71 \pm 4.56$, respectively; $\mathrm{P}=0.006$, Table 1).

The diameter of MPA and RPA in the rTOF group was significantly larger than that in the control group (19.74 \pm 4.01 vs. $14.97 \pm 2.37$ for MPA, $\mathrm{P}=0.001 ; 12.04 \pm 3.28$ vs. $8.99 \pm 1.23$ for $\mathrm{RPA}, \mathrm{P}=0.004$, respectively, Table 2). The peak velocities of LPA in the rTOF group and control group were significantly different $(151.76 \pm 32.2 v s$. $116.3 \pm 20.64$, respectively; $\mathrm{P}=0.002$, Table 2). However, the net forward flow showed no significant difference between groups $(51.7 \pm 21.26$ vs. $44.26 \pm 9.72$ for $\mathrm{MPA}, \mathrm{P}=0.558$; $30.22 \pm 14.46$ vs. $23.97 \pm 4.16$ for $\mathrm{RPA}, \mathrm{P}=0.392 ; 20.89 \pm 13.17$ vs. $19.34 \pm 5.32$ for LPA, $\mathrm{P}=0.602$ respectively, Table 2).

The mean regurgitation fraction of MPA, RPA, and LPA in the rTOF group was $31 \%( \pm 17 \%), 26 \%( \pm 16 \%)$, and $30 \%( \pm 15 \%)$, respectively (Table 3$)$. 
Table 1 CMR measurements in patients and healthy volunteers (mean \pm SD)

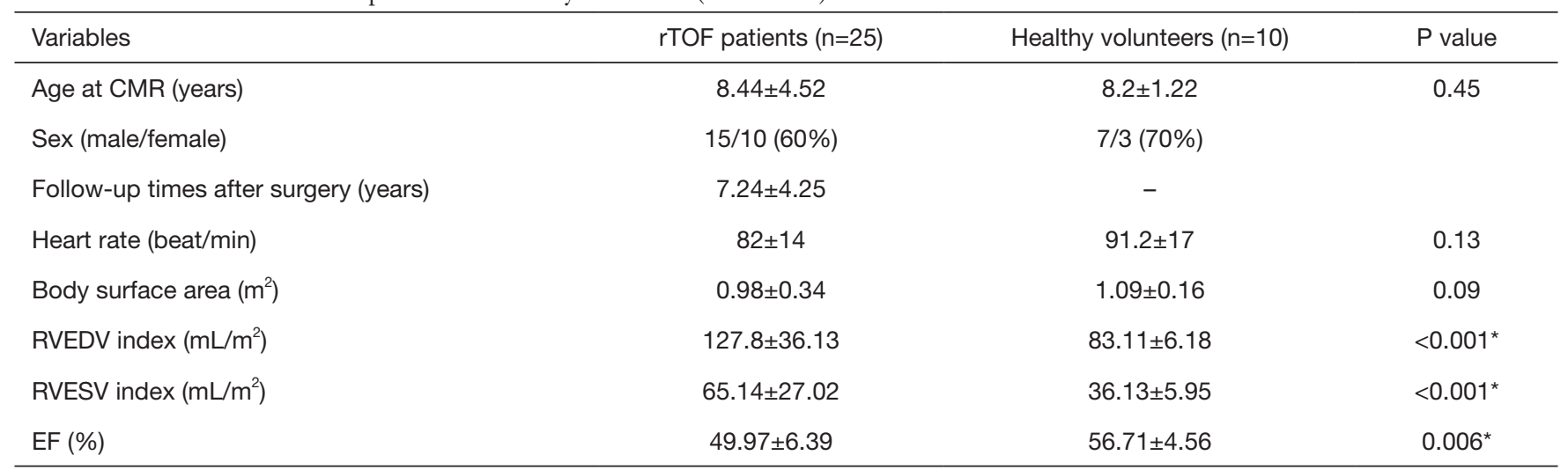

Data are expressed as mean \pm standard deviation or $\mathrm{n}(\%) .{ }^{*}$, represents a statistically significant difference. rTOF, repaired tetralogy of Fallot; EF, ejection fraction; RVEDV, right ventricular end-diastole volume; RVESV, right ventricular end-systolic volume.

Table 2 Pulmonary artery systolic diameter and blood flow parameters in rTOF patients and healthy controls

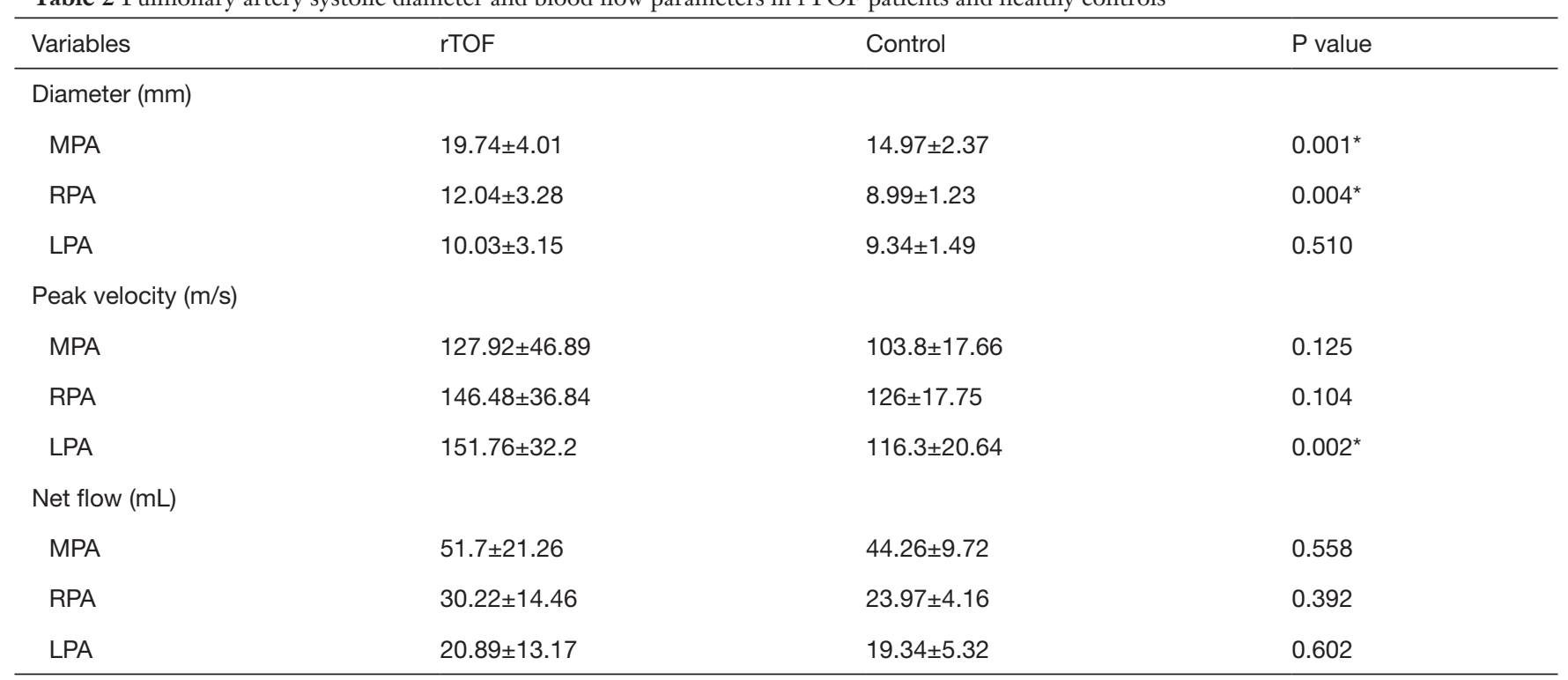

Data are expressed as mean \pm standard deviation. ${ }^{*}$, represents a statistically significant difference. MPA, main pulmonary artery; RPA, right pulmonary artery; LPA, left pulmonary artery.

\section{Flow patterns}

Velocity vector and streamline visualization were successfully performed in all subjects. The healthy child volunteers exhibited normal uniform and laminar flow patterns in the MPA and LPA in 10 cases and in the RPA in 9 cases. In 1 abnormal case of RPA flow, grade 1 helical flow was observed. Furthermore, 7 of 25 rTOF subjects had helical flow patterns in the RPA (3 cases of grade 1, 4 cases of grade 2), and 1 subject had helical flow patterns in the LPA (1 case of grade 1 ). Meanwhile, 14 of 25 rTOF subjects had vortex flow patterns in the MPA (12 cases of grade 1, 2 cases of grade 2), while 1 case had vortex flow patterns in the RPA (1 case of grade 1 ), and 2 cases had them in the LPA (2 cases of grade 1$)$. The flow grading was as follows: group 1 included 6 rTOF patients; group 2 included 9 rTOF patients; group 3 included 10 rTOF patients; group 4 included 9 child volunteers.

\section{Correlation analysis}

The correlated peak WSS, maximum peak systolic EL, and 
Table 3 Comparison of peak systolic velocity, regurgitation fraction, and flow grading in rTOF patients and healthy controls

\begin{tabular}{|c|c|c|c|c|c|c|c|}
\hline \multirow{3}{*}{ Subject } & \multirow{3}{*}{ Regurgitation fraction (\%) } & \multicolumn{6}{|c|}{ Flow grading } \\
\hline & & \multicolumn{3}{|c|}{ Helix (cases) } & \multicolumn{3}{|c|}{ Vortex (cases) } \\
\hline & & 0 & 1 & 2 & 0 & 1 & 2 \\
\hline \multicolumn{8}{|l|}{ rTOF } \\
\hline MPA & $31 \pm 17$ & 25 & - & - & 11 & 12 & 2 \\
\hline LPA & $30 \pm 15$ & 24 & 1 & - & 23 & 2 & - \\
\hline \multicolumn{8}{|l|}{ Control } \\
\hline MPA & 0 & 10 & - & - & 10 & - & - \\
\hline RPA & 0 & 9 & 1 & - & 10 & - & - \\
\hline
\end{tabular}

The median flow grading with ranges (none $=0$, moderate $=1$, severe $=2$ ) in the MPA, LPA, and RPA. Group 1: the rTOF cases of severe (grade 2) flow grading, including the MPA, RPA, and LPA. Group 2: the rTOF cases of mild (grade 1) flow grading, including the MPA, RPA, and LPA. Group 3: the rTOF cases of none flow grading, including the MPA, RPA, and LPA. Group 4: none-flow grading in controls. MPA, main pulmonary artery; RPA, right pulmonary artery; LPA, left pulmonary artery; rTOF, repaired tetralogy of Fallot; MIP, maximum intensity plot; ROI, regions of interest.

cardiac function were measured in the MPA, RPA, and LPA of the patient and control groups (Table 4). In the MPA, there was a correlation between peak WSS and $\mathrm{PR}(\mathrm{R}=0.48$, $\mathrm{P}=0.014)$. In the RPA, there was a correlation between peak systolic EL and RVEDV ( $\mathrm{R}=0.51, \mathrm{P}=0.008)$, and between peak systolic EL and RVESV ( $\mathrm{R}=0.51, \mathrm{P}=0.009)$.

\section{Quantitative flow grading analysis}

Results of the quantitative flow grading analysis of MPA, RPA, and LPA are summarized in Table 5. Peak WSS and peak EL of flow the grading groups during the cardiac cycle in the MPA, RPA, and LPA with rTOF and healthy controls are shown in Figure 4. The peak systole and diastole WSS of group $1+2$ were significantly different from those of group $3+4$ in the MPA $(\mathrm{P}<0.05$, Figure 5$)$. The peak systole and diastole EL of group $1+2$ was significantly different from group 3+4 in the MPA (respectively; $\mathrm{P}<0.05$, Figure 5). The peak systole EL of group $1+2$ was significantly different from group $3+4$ in the RPA $(\mathrm{P}<0.01$, Figure 5$)$.

\section{Discussion}

TOF patients following surgical repair represent a growing population with congenital heart disease, as they now survive into adulthood. Progressive RV enlargement is common in rTOF patients with moderate or severe PR (17). In recent years, CMR has been used to assess cardiac function, and is considered the gold standard for the quantification of RV function (18). Our results demonstrated that the $\mathrm{EDV}_{\mathrm{i}}, \mathrm{ESV}_{\mathrm{i}}$, and $\mathrm{EF}$ were significantly different in rTOF patients compared to healthy volunteers after a follow-up $7.24 \pm 4.25$ years. To better guide treatment, understanding the abnormal pulmonary arteries hemodynamics and how they impact RV function has become increasingly important. 4D flow CMR has been widely used to measure non-invasively complex 3D hemodynamic changes in the pulmonary arteries (19). François et al. (1) observed increased PA WSS values in rTOF subjects compared with healthy controls. Our study was to assess the flow grading of the helix and vortex in pulmonary artery hemodynamics and to explore the relationship between pulmonary hemodynamic changes and right heart function.

\section{Vortex flow patterns in rTOF}

We found that most of the vortices existed in the MPA during systole and diastole (Table 3). Vortices might be the key flow characteristic of a dilated pulmonary artery (20). In our study, the MPA and RPA diameters in the rTOF group were significantly larger than those in the control 
Table 4 Comparisons of the correlations between hemodynamic parameters and the routine CMR measurements in rTOF patients and healthy controls

\begin{tabular}{|c|c|c|c|c|c|c|c|c|}
\hline Variables & \multicolumn{2}{|c|}{ RVEDVi } & \multicolumn{2}{|c|}{ RVESVi } & \multicolumn{2}{|c|}{ RVEF } & \multicolumn{2}{|c|}{ MPA PR } \\
\hline \multicolumn{9}{|l|}{ rTOF } \\
\hline Wall shear stress ${ }_{\mathrm{MPA}}$ & -0.06 & 0.746 & -0.16 & 0.42 & 0.13 & 0.524 & $0.483^{*}$ & $0.014^{*}$ \\
\hline Energy loss $_{\mathrm{MPA}}$ & 0.28 & 0.169 & 0.28 & 0.17 & -0.19 & 0.364 & 0.333 & 0.103 \\
\hline Energy loss $\mathrm{RPA}_{\mathrm{RA}}$ & $0.51^{*}$ & $0.008^{*}$ & $0.51^{\star}$ & $0.009^{*}$ & -0.23 & 0.260 & 0.303 & 0.141 \\
\hline Wall shear stress ${ }_{\mathrm{LPA}}$ & -0.06 & 0.76 & -0.12 & 0.55 & 0.152 & 0.466 & 0.226 & 0.275 \\
\hline Energy loss $_{\mathrm{LPA}}$ & 0.29 & 0.156 & 0.22 & 0.289 & 0.015 & 0.943 & 0.136 & 0.516 \\
\hline \multicolumn{9}{|l|}{ Control } \\
\hline Wall shear stress $\mathrm{RPA}_{\mathrm{RA}}$ & 0.20 & 0575 & -0.03 & 0.93 & 0.157 & 0.665 & - & - \\
\hline Energy loss RPA $_{\text {f }}$ & -0.027 & 0.938 & -0.08 & 0.81 & 0.139 & 0.701 & - & - \\
\hline Wall shear stress $\mathrm{LPA}_{\mathrm{LA}}$ & 0.105 & 0.772 & -0.09 & 0.79 & 0.23 & 0.522 & - & - \\
\hline Energy loss $_{\mathrm{LPA}}$ & -0.187 & 0.603 & -0.21 & 0.55 & 0.222 & 0.537 & - & - \\
\hline
\end{tabular}

*, represents a statistically significant difference. RVEDV, right ventricular end-diastole volume; RVESV, right ventricular end-systole volume; RVEF, right ventricular ejection fraction; MPA PR, main pulmonary artery regurgitation fraction.

group. This concept was confirmed by previous 4D flow studies of the pulmonary artery, which showed that vortices and helices were associated with increased WSS in the pulmonary artery wall, suggesting that flow was implicated in artery dilatation and that a change to the artery wall composition might be a secondary phenomenon $(21,22)$.

\section{Helical flow patterns in rTOF}

In our study, most of the helical flow existed in the RPA during systole and diastole (Table 3). Helical flow can be regarded as the rotational motion of blood oriented normally to the mainstream of blood flow during its passage through the artery. In the helical flow, the streamlines in the lumen of the arteries demonstrate a helical shape, and those on the cross-sections of the arteries exhibit secondary rotations. Stonebridge et al. stated that when compared with typical flow patterns, helical flow may be more efficient and require less energy to drive blood through the tapering and branching arterial system (23).

Moreover, the increased helical flow induced by pulsatile blood flow in the thoracic aorta and coronary artery would further enhance the oxygen transport (24). Liu $e t$ al. reported that at the same flow rate, the helical flow in the MPA enhanced flow mixing and the WSS (25). The occurrence of helical flow in the RPA was largely the result of complex geometries (26) and pulmonary vascular elastance. However, the mechanism is not well understood.

\section{Subgroup analysis of flow grading}

In our study, systole and diastole WSS were statistically different between group $1+2$ (moderate and severe flow grading rTOF cases) and group 3+4 (none-flow grading rTOF cases and controls) in the MPA. These results indicated that changes in hemodynamics already existed in the MPA as evidenced by the severity of the helix and vortex when RVEF was normal.

The peak systole EL of group 1+2 was significantly different from that of group $3+4$ in the MPA and RPA. EL was calculated by secondary non-turbulent flow and was sensitive to the presence of large-scale vortices and helices 
Table 5 Summary of quantitative flow grading analysis between group 1+2 and group 3+4

\begin{tabular}{|c|c|c|c|}
\hline Parameter & Group 1 + Group 2 & Group 3 + Group 4 & $P$ \\
\hline RVEDVi $\left(\mathrm{mL} / \mathrm{m}^{2}\right)$ & $130.98 \pm 42.51$ & $104.06 \pm 27.48$ & 0.060 \\
\hline RVESVi $\left(\mathrm{mL} / \mathrm{m}^{2}\right)$ & $66.32 \pm 32.73$ & $50.44 \pm 18.75$ & 0.146 \\
\hline RVEF (\%) & $50.65 \pm 6.23$ & $53.69 \pm 5.88$ & 0.435 \\
\hline \multicolumn{4}{|l|}{ Systole } \\
\hline Wall shear stress $\mathrm{MPA}_{\mathrm{MP}}$ & $0.24 \pm 0.09$ & $0.19 \pm 0.04$ & $0.026^{*}$ \\
\hline Energy loss $_{\mathrm{MPA}}$ & $33.14 \pm 12.43$ & $21.17 \pm 8.22$ & $0.002^{* *}$ \\
\hline Wall shear stress $\mathrm{RPA}_{\mathrm{RP}}$ & $0.44 \pm 0.18$ & $0.56 \pm 0.21$ & 0.086 \\
\hline Energy loss ${ }_{\mathrm{LPA}}$ & $16.57 \pm 5.43$ & $16.67 \pm 8.44$ & 0.468 \\
\hline \multicolumn{4}{|l|}{ Diastole } \\
\hline Wall shear stress ${ }_{\mathrm{MPA}}$ & $0.13 \pm 0.09$ & $0.08 \pm 0.04$ & $0.021^{*}$ \\
\hline Energy loss MPA & $15.87 \pm 10.49$ & $8.54 \pm 4.99$ & $0.011^{*}$ \\
\hline Wall shear stress ${ }_{\text {RPA }}$ & $0.15 \pm 0.08$ & $0.14 \pm 0.07$ & 0.791 \\
\hline Energy loss RPA & $9.06 \pm 9.27$ & $6.02 \pm 4.36$ & 0.992 \\
\hline Wall shear stress ${ }_{\mathrm{LPA}}$ & $0.17 \pm 0.08$ & $0.18 \pm 0.11$ & 0.791 \\
\hline Energy loss $_{\mathrm{LPA}}$ & $4.11 \pm 3.63$ & $5.14 \pm 3.77$ & 0.187 \\
\hline
\end{tabular}

MPA, main pulmonary artery; RPA, right pulmonary artery; LPA, left pulmonary artery. Asterisk(s) represent a statistically significant difference: *, indicates statistical significance $(\mathrm{P}<0.05)$; ${ }^{* *}$, indicates statistical significance $(\mathrm{P}<0.01)$.

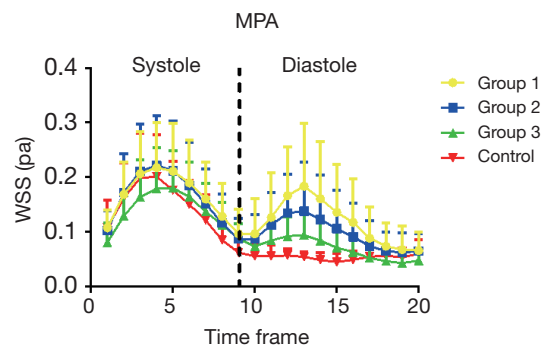

MPA
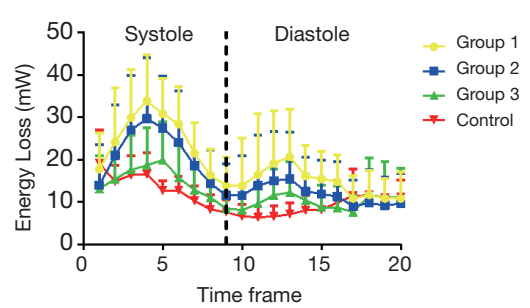

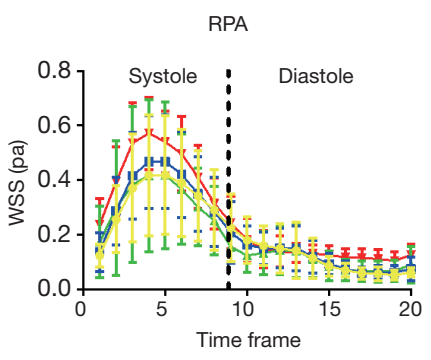

RPA

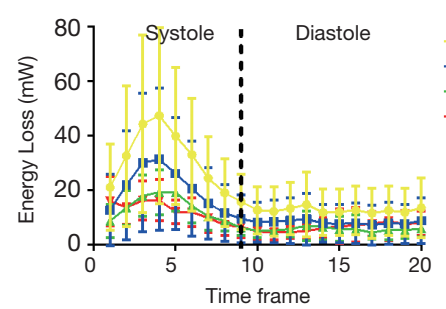

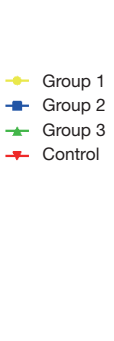

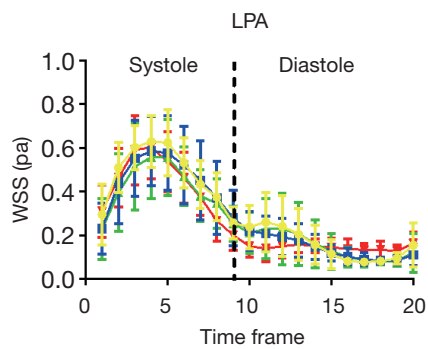

LPA
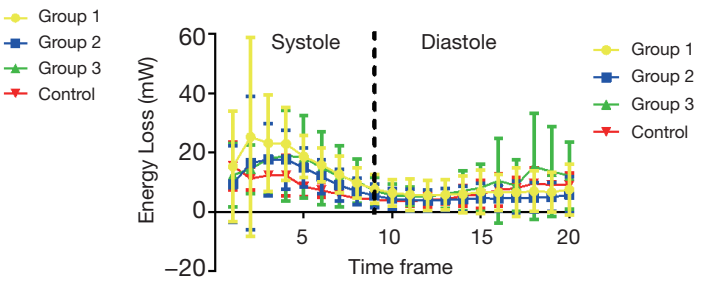

Figure 4 Peak WSS and peak EL of the flow grading group during the cardiac cycle in the MPA, RPA, and LPA of rTOF and volunteers. MPA, main pulmonary artery; RPA, right pulmonary artery; LPA, left pulmonary artery; WSS, wall shear stress; EL, energy loss. 

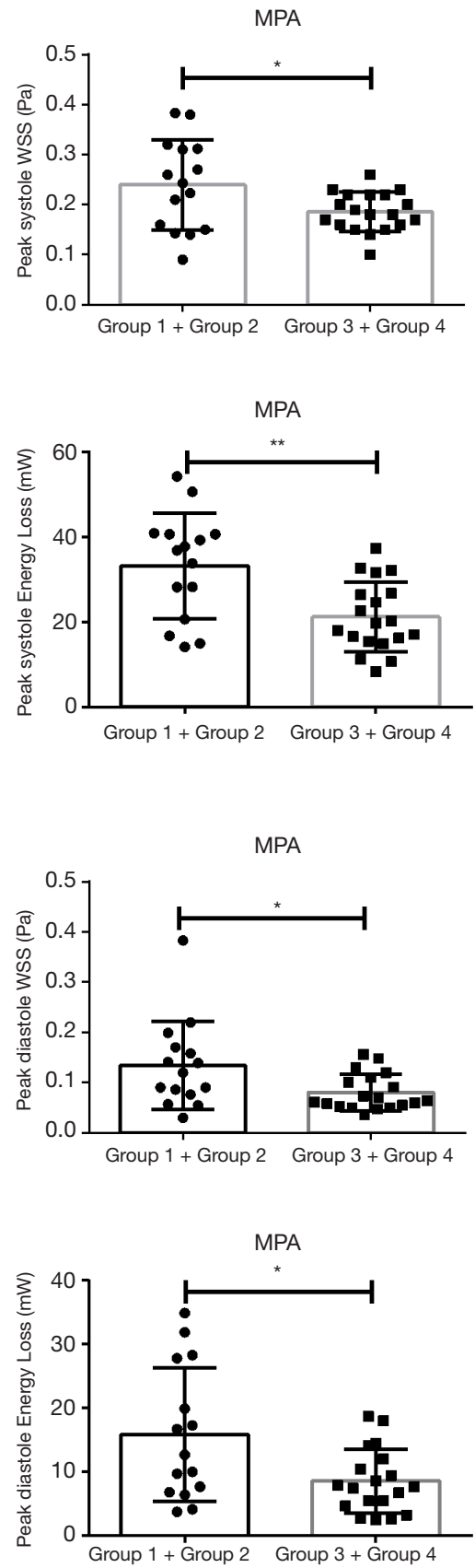

RPA

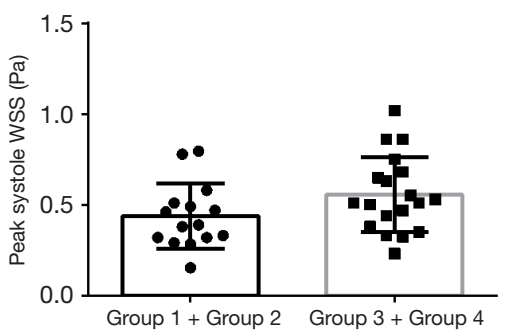

RPA

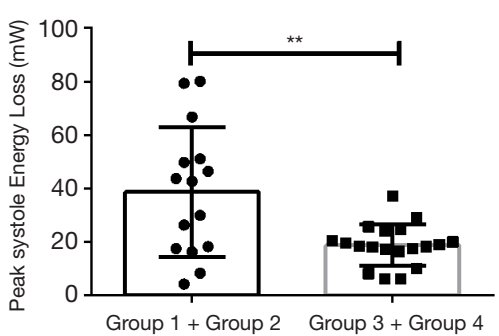

RPA

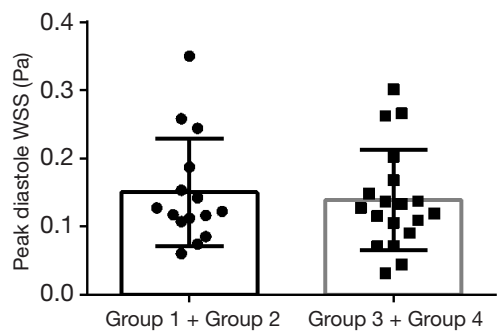

RPA

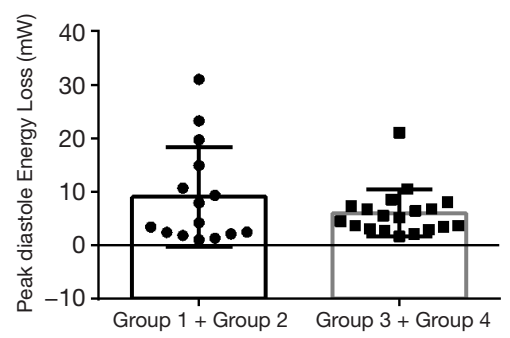

LPA

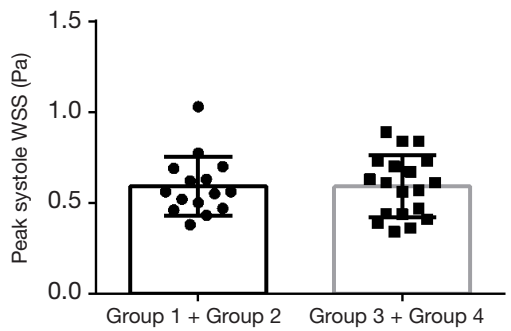

LPA

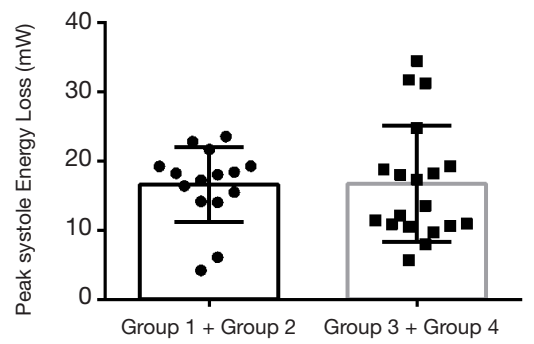

LPA

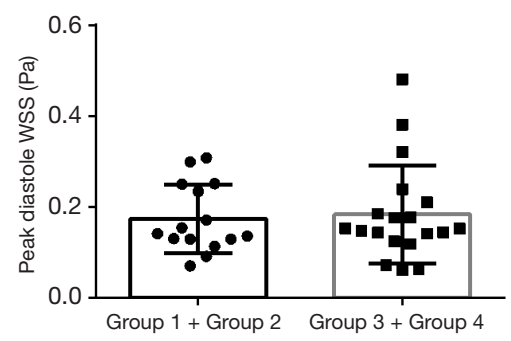

LPA

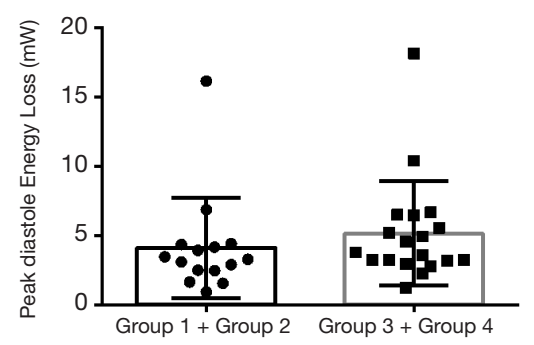

Figure 5 Comparison of cardiac function, WSS, and EL in group $1+2$ and group $3+4$ during the systole and diastole in the MPA, RPA, and LPA of rTOF patients and volunteers. *, indicates statistical significance $(\mathrm{P}<0.05)$; ${ }^{* *}$, indicates statistical significance $(\mathrm{P}<0.01)$. MPA, main pulmonary artery; RPA, right pulmonary artery; LPA, left pulmonary artery; WSS, wall shear stress; EL, energy loss; rTOF, repaired tetralogy of Fallot. 
in our study (27). Our explanation for this was that peak EL is a component of mechanically produced kinetic energy, and kinetic energy effectively introduces added work on the RV and ultimately elevates the afterload (28).

\section{The relationship between bemodynamic parameters and $R V$ function}

In our study, there were correlations between peak WSS and PR in the MPA. Geva et al. reported that chronic PR leads to ventricular deterioration (29). PR may play a role in the formation of vortex flow in MPA, although the etiology is still poorly understood. In addition, flow vortices have been associated with alteration in WSS and affect endothelial function. These were consistent with our findings.

Earlier studies reported abnormal RV-PA coupling with normal RV EF in the rTOF group. A dramatically increased EL noted in RPA with more severe RV enlargement was considered evidence of impaired ventricular-vascular coupling (30). In our study, there were correlations between peak systolic EL and RVEDV $\mathrm{i}_{\mathrm{i}} / \mathrm{RVESVi}$ in the RPA. These inefficiencies changed performance such as in the areas of pulmonary vascular reserve, ventricular remodeling, and decreased performance (31). Our study supports the possibility that hemodynamic changes are associated with ventricular deterioration and may be helpful in understanding how PA-RV interactions impact RV function.

\section{Limitations}

Some limitations to our study should addressed. First, our results were inherently limited by the small sample size of a pilot study. Second, the limited spatial resolution might have also masked details of the velocity gradients, due to the limited scanning time. Third, 4D flow data sets were acquired with prospective ECG-gating during freebreathing. A validation study showed good agreement in hemodynamic change during scans with and without respiratory gating (32), but prospective gating might have influenced backward flow and regurgitation fraction values. Fourth, irregular, and rapid fluctuations due to turbulence were not directly represented in the three-directional blood flow velocities in each imaging voxel as measured by the 4D flow MRI (33). Thus, the severe stenosis of pulmonary artery cases was not included in the study. Fifth, the current spatiotemporal resolution of 4D flow MRI and the smoothing kernel used for the EL quantification might have underestimated the magnitude of the laminar component of viscous EL (34). Finally, it is essential to note that we analyzed WSS and EL only at peak systolic and diastolic time points; this is a limitation in our workflow. In future work, we expect to assess a larger cohort of rTOF patients, improve stratification of flow phenomena, and provide practical guidance regarding management.

\section{Conclusions}

Increased peak WSS and EL were associated with pulmonary hemodynamic changes in the MPA and RPA. These might be an earlier marker of evolving hemodynamic inefficiency than traditional parameters. We hope that a more comprehensive understanding of pulmonary artery hemodynamics in rTOF might lead to greater insight into PA-RV interactions and how they impact RV function.

\section{Acknowledgments}

Funding: The present study was funded by the National Key R\&D Program of China (No. 2018YFB1107100), the Shanghai Committee of Science and Technology (No. 17411965400 and 17DZ2253100), Shanghai Municipal Commission of Health and Family Planning (No. 201740095) and the Shanghai Rising Stars of Medical Talent Medical Imaging Practitioner Program.

\section{Footnote}

Conflicts of Interest: All authors have completed the ICMJE uniform disclosure form (available at http://dx.doi. org/10.21037/qims.2020.03.23). The authors have no conflicts of interest to declare.

Ethical Statement: This single-center retrospective cohort study was approved by the hospital institutional review board. Informed consent was signed by the participant's parents or legal guardians.

Open Access Statement: This is an Open Access article distributed in accordance with the Creative Commons Attribution-NonCommercial-NoDerivs 4.0 International License (CC BY-NC-ND 4.0), which permits the noncommercial replication and distribution of the article with the strict proviso that no changes or edits are made and the original work is properly cited (including links to both the 
formal publication through the relevant DOI and the license). See: https://creativecommons.org/licenses/by-nc-nd/4.0/.

\section{References}

1. François CJ, Srinivasan S, Schiebler ML, Reeder SB, Niespodzany E, Landgraf BR, Wieben O, Frydrychowicz A. 4D cardiovascular magnetic resonance velocity mapping of alterations of right heart flow patterns and main pulmonary artery hemodynamics in tetralogy of Fallot. J Cardiovasc Magn Reson 2012;14:16.

2. Al Habib HF, Jacobs JP, Mavroudis C, Tchervenkov CI, O'Brien SM, Mohammadi S, Jacobs ML. Contemporary patterns of management of tetralogy of Fallot: data from the Society of Thoracic Surgeons Database. Ann Thorac Surg 2010;90:813-9; discussion 819-20.

3. Bacha EA, Scheule AM, Zurakowski D, Erickson LC, Hung J, Lang P, Mayer JE Jr, del Nido PJ, Jonas RA. Long-term results after early primary repair of tetralogy of Fallot. J Thorac Cardiovasc Surg 2001;122:154-61.

4. van Ooij P, Potters WV, Collins J, Carr M, Carr J, Malaisrie SC, Fedak PW, McCarthy PM, Markl M, Barker AJ. Characterization of abnormal wall shear stress using 4D flow MRI in human bicuspid aortopathy. Ann Biomed Eng 2015;43:1385-97.

5. Fredriksson A, Trzebiatowska-Krzynska A, Dyverfeldt P, Engvall J, Ebbers T, Carlhäll CJ. Turbulent kinetic energy in the right ventricle: Potential MR marker for risk stratification of adults with repaired Tetralogy of Fallot. J Magn Reson Imaging 2018;47:1043-53.

6. Barker AJ, van Ooij P, Bandi K, Garcia J, Albaghdadi M, McCarthy P, Bonow RO, Carr J, Collins J, Malaisrie SC, Markl M. Viscous energy loss in the presence of abnormal aortic flow. Magn Reson Med 2014;72:620-8.

7. Jeong D, Anagnostopoulos PV, Roldan-Alzate A, Srinivasan S, Schiebler ML, Wieben O, François CJ. Ventricular kinetic energy may provide a novel noninvasive way to assess ventricular performance in patients with repaired tetralogy of Fallot. J Thorac Cardiovasc Surg 2015;149:1339-47.

8. Sjöberg P, Bidhult S, Bock J, Heiberg E, Arheden H, Gustafsson R, Nozohoor S, Carlsson M. Disturbed left and right ventricular kinetic energy in patients with repaired tetralogy of Fallot: pathophysiological insights using 4D-flow MRI. Eur Radiol 2018;28:4066-76.

9. Geiger J, Markl M, Jung B, Grohmann J, Stiller B, Langer M, Arnold R. 4D-MR flow analysis in patients after repair for tetralogy of Fallot. Eur Radiol 2011;21:1651-7.
10. van der Hulst AE, Westenberg JJ, Kroft LJ, Bax JJ, Blom NA, de Roos A, Roest AA. Tetralogy of fallot: 3D velocityencoded MR imaging for evaluation of right ventricular valve flow and diastolic function in patients after correction. Radiology 2010;256:724-34.

11. Hsiao A, Alley MT, Massaband P, Herfkens RJ, Chan FP, Vasanawala SS. Improved cardiovascular flow quantification with time-resolved volumetric phasecontrast MRI. Pediatr Radiol 2011;41:711-20.

12. Dyverfeldt P, Bissell M, Barker AJ, Bolger AF, Carlhäll CJ, Ebbers T, Francios CJ, Frydrychowicz A, Geiger J, Giese D, Hope MD, Kilner PJ, Kozerke S, Myerson S, Neubauer S, Wieben O, Markl M. 4D flow cardiovascular magnetic resonance consensus statement. J Cardiovasc Magn Reson 2015;17:72.

13. Zhong L, Schrauben EM, Garcia J, Uribe S, Grieve SM, Elbaz MSM, Barker AJ, Geiger J, Nordmeyer S, Marsden A, Carlsson M, Tan RS, Garg P, Westenberg JJM, Markl M, Ebbers T. Intracardiac 4D Flow MRI in Congenital Heart Disease: Recommendations on Behalf of the ISMRM Flow \&amp; Motion Study Group. J Magn Reson Imaging 2019;50:677-81.

14. Bürk J, Blanke P, Stankovic Z, Barker A, Russe M, Geiger J, Frydrychowicz A, Langer M, Markl M. Evaluation of $3 \mathrm{D}$ blood flow patterns and wall shear stress in the normal and dilated thoracic aorta using flow-sensitive 4D CMR. J Cardiovasc Magn Reson 2012;14:84.

15. Kilner PJ, Yang GZ, Mohiaddin RH, Firmin DN, Longmore DB. Helical and retrograde secondary flow patterns in the aortic arch studied by three-directional magnetic resonance velocity mapping. Circulation 1993;88:2235-47.

16. Stalder AF, Russe MF, Frydrychowicz A, Bock J, Hennig J, Markl M. Quantitative 2D and 3D phase contrast MRI: optimized analysis of blood flow and vessel wall parameters. Magn Reson Med 2008;60:1218-31.

17. Gatzoulis MA, Balaji S, Webber SA, Siu SC, Hokanson JS, Poile C, Rosenthal M, Nakazawa M, Moller JH, Gillette PC, Webb GD, Redington AN. Risk factors for arrhythmia and sudden cardiac death late after repair of tetralogy of Fallot: a multicentre study. Lancet 2000;356:975-81.

18. Catalano O, Antonaci S, Opasich C, Moro G, Mussida M, Perotti M, Calsamiglia G, Frascaroli M, Baldi M, Cobelli F. Intra-observer and interobserver reproducibility of right ventricle volumes, function and mass by cardiac magnetic resonance. J Cardiovasc Med (Hagerstown) 2007;8:807-14.

19. Markl M, Frydrychowicz A, Kozerke S, Hope M, 
Wieben O. 4D flow MRI. J Magn Reson Imaging 2012;36:1015-36.

20. Meierhofer C, Schneider EP, Lyko C, Hutter A, Martinoff S, Markl M, Hager A, Hess J, Stern H, Fratz S. Wall shear stress and flow patterns in the ascending aorta in patients with bicuspid aortic valves differ significantly from tricuspid aortic valves: a prospective study. Eur Heart J Cardiovasc Imaging 2013;14:797-804.

21. Bissell MM, Loudon M, Neubauer S, Myerson SG. Abnormal Haemodynamic Flow Patterns in Bicuspid Pulmonary Valve Disease. Front Physiol 2017;8:374.

22. Guzzardi DG, Barker AJ, van Ooij P, Malaisrie SC, Puthumana JJ, Belke DD, Mewhort HE, Svystonyuk DA, Kang S, Verma S, Collins J, Carr J, Bonow RO, Markl M, Thomas JD, McCarthy PM, Fedak PW. Valve-Related Hemodynamics Mediate Human Bicuspid Aortopathy: Insights From Wall Shear Stress Mapping. J Am Coll Cardiol 2015;66:892-900.

23. Stonebridge PA, Brophy CM. Spiral laminar flow in arteries? Lancet 1991;338:1360-1.

24. Coppola G, Caro C. Arterial geometry, flow pattern, wall shear and mass transport: potential physiological significance. J R Soc Interface 2009;6:519-28.

25. Liu X, Sun A, Fan Y, Deng X. Physiological significance of helical flow in the arterial system and its potential clinical applications. Ann Biomed Eng 2015;43:3-15.

26. Acevedo-Bolton G, Amans MR, Kefayati S, Halbach $\mathrm{V}$, Saloner D. Four dimensional magnetic resonance velocimetry for complex flow in the jugular vein. Quant Imaging Med Surg 2015;5:635-7.

27. Cibis M, Jarvis K, Markl M, Rose M, Rigsby C, Barker AJ, Wentzel JJ. The effect of resolution on viscous dissipation measured with 4D flow MRI in patients with Fontan circulation: Evaluation using computational fluid

Cite this article as: $\mathrm{Hu} \mathrm{L}$, Ouyang $\mathrm{R}$, Sun A, Wang Q, Guo C, Peng Y, Qin Y, Zhang Y, Xiang Y, Zhong Y. Pulmonary artery hemodynamic assessment of blood flow characteristics in repaired tetralogy of Fallot patients versus healthy child volunteers. Quant Imaging Med Surg 2020;10(5):921-933. doi: 10.21037/qims.2020.03.23 dynamics. J Biomech 2015;48:2984-9.

28. Schäfer M, Barker AJ, Jaggers J, Morgan GJ, Stone ML, Truong U, Browne LP, Malone L, Ivy DD, Mitchell MB. Abnormal aortic flow conduction is associated with increased viscous energy loss in patients with repaired tetralogy of Fallot. Eur J Cardiothorac Surg 2020;57:588-95.

29. Geva T. Indications for pulmonary valve replacement in repaired tetralogy of fallot: the quest continues. Circulation 2013;128:1855-7.

30. Egbe AC, Kothapalli S, Miranda WR, Pislaru S, Ammash NM, Borlaug BA, Pellikka PA, Najam M, Connolly HM. Assessment of Right Ventricular-Pulmonary Arterial Coupling in Chronic Pulmonary Regurgitation. Can J Cardiol 2019;35:914-22.

31. Warmerdam E, Krings GJ, Leiner T, Grotenhuis HB. Three-dimensional and four-dimensional flow assessment in congenital heart disease. Heart 2020;106:421-6.

32. Hsiao A, Yousaf U, Alley MT, Lustig M, Chan FP, Newman B, Vasanawala SS. Improved quantification and mapping of anomalous pulmonary venous flow with fourdimensional phase-contrast MRI and interactive streamline rendering. J Magn Reson Imaging 2015;42:1765-76.

33. Barker AJ, Staehle F, Bock J, Jung BA, Markl M. Analysis of complex cardiovascular flow with threecomponent acceleration-encoded MRI. Magn Reson Med 2012;67:50-61.

34. Töger J, Arvidsson PM, Bock J, Kanski M, Pedrizzetti G, Carlsson M, Arheden H, Heiberg E. Hemodynamic forces in the left and right ventricles of the human heart using 4D flow magnetic resonance imaging: Phantom validation, reproducibility, sensitivity to respiratory gating and free analysis software. PLoS One 2018;13:e0195597. 